\title{
Propuesta metodológica para la integración de un sistema de gestión documental basado en los referenciales NTC ISO 9001, NTCGP 1000 e ISO/IEC 17025
}

\author{
Methodological proposal for the integration of a document \\ management system based on NTC ISO 9001, NTC GP1000 \\ and ISO / IEC 17025
}

Recibido: 29 de agosto de 2016 Revisado: 30 de septiembre de 2016 Aceptado: 2 de diciembre de 2016

Yanneth Garcia Melo***

Instituto Colombiano Agropecuario, Laboratorio Nacional de Diagnostico Veterinario

Nancy Carolina Suarez Católico****

Vicar Farmacéutica S.A

* Artículo de resultado de investigación.

** Bacterióloga y Laboratorista Clínica; especialista en Banco de Sangre y magíster en Calidad y Gestión Integral. Responsable analítico del Laboratorio de Diagnostico Aviar en el Laboratorio Nacional de Diagnostico Veterinario (LNDV), perteneciente al Instituto Colombiano Agropecuario (ICA).Correo electrónico: yanneth.garcia@ica.gov.co; yanneth.garcia77@gmail.com.co

*** Bacterióloga y Laboratorista Clínica y magíster en Calidad y Gestión Integral. Analista de microbiología en el Laboratorio de Control de Calidad de Vicar Farmacéutica S.A. Correo electrónico: Nancysuarez@santotomas.edu.co carito.s23@hotmail.com 


\section{RESUMEN}

El presente estudio se enfoca en identificar los aspectos críticos de la gestión documental del Laboratorio Nacional de Diagnostico Veterinario (LNDV), perteneciente al Instituto Colombiano Agropecuario (ICA), con respecto a los referenciales NTC ISO 9001, NTCGP 1000 e ISO/IEC 17025. Asimismo reconoce los modelos de gestión que permitan su integración para fortalecer la gestión documental de la entidad y estructura una propuesta metodológica de integración durante el periodo 2014-2016. La importancia de esta investigación se enmarca en la generación de nuevo conocimiento sobre modelos de integración de sistemas de gestión a partir de un estudio cualitativo, en el cual se llega a la conclusión de que los aspectos críticos encontrados ocasionan falencias en la ejecución de la gestión documental que pertenece a los diferentes procesos estructurales de los sistemas de gestión. Las causas y consecuencias de estos últimos están asociadas a componentes normativos como conocimiento, formación y toma de conciencia, normas y leyes, planificación, responsabilidad y autoridad, gestión por procesos, recursos para el sostenimiento de los sistemas de gestión, procedimiento control de documentos e implementación. Si uno de estos componentes presenta falencias en el enfoque basado en procesos, propuesto por los sistemas de gestión con los cuales la entidad está certificada, las causas y consecuencias de estas estarán reflejadas en cada una de las interacciones y jerarquías funcionales de la entidad. Como producto final se elaboró una propuesta metodológica, basada en la estructura de alto nivel, como método para su construcción y se espera que permita la integración de los sistemas de gestión en estudio.

Palabras clave: estructura de alto nivel, gestión documental, integración de sistemas de gestión.

\section{ABSTRACT}

This study emphasizes the identification of the critical aspects of the documentary management of the National Laboratory of Veterinary Diagnosis (LNDV) belonging to the Colombian Agricultural Institute (ICA). with respect to the references NTC ISO 9001, NTCGP 1000 and ISO / IEC 17025, identifying the management models that allow its integration to strengthen the documentary management of the entity and structuring a methodological proposal of integration during the period from 2014 to 2016. The importance of this research is framed in the generation of new knowledge on models of integration of management systems from a qualitative study, in which it is concluded that the critical aspects found cause failures in the executions that correspond to the documentary management and belong to the different structural processes of the management systems, in which its causes and consequences are associated to the following normative components: knowledge, training and awareness, standards and laws, planning, responsibility and authority, process management, resources for sustenance management systems, document control procedures and implementation. That is to say, if one of these components has shortcomings in the process-based approach, proposed by the management systems with which the entity is certified, the causes and consequences of these are reflected in each of the interactions and functional hierarchies of the entity. The final product was elaborated a methodological proposal, which is based on the high-level structure as a method for its construction and is expected to allow the integration of management systems under study.

Keywords: High-level structure, document management, Integration of management systems. 


\section{INTRODUCCIÓN}

En los últimos años el Instituto Colombiano Agropecuario (ICA) se ha encaminado a un proceso de cambio que lo reafirme como un verdadero socio estratégico del agronegocio colombiano, y para lograrlo uno de los proyectos formulados busca la implementación de modelos de gestión orientados a diferentes propósitos, entre los cuales están los modelos de gestión de la calidad ISO 9001 y NTCGP 1000, que han demostrado facilitar la mejora continua en el cumplimiento de requisitos de la sociedad, ejecución de planes de acción y seguimiento que contribuyen con el control interno como lo mencionan Villaquirán y Nieto (2016). La Subgerencia de análisis y diagnóstico ha implementado el modelo ISO/IEC 17025:2005, que contiene requisitos generales para la competencia de los laboratorios de ensayo y calibración que deseen demostrar que son técnicamente competentes y capaces de generar resultados válidos.

En la actualidad en el Laboratorio Nacional de Diagnostico Veterinario (LNDV) del ICA se presentan falencias en las ejecuciones que corresponden a la gestión documental, entre las cuales están la falta de divulgación de nuevas normatividades, la actualización del sistema de gestión de calidad, la existencia de un subsistema de control documental en el LNDV, diferente al de la institución en los requerimientos que surgen para dar cumplimiento a la parte legal y normativa y la no evidencia del funcionamiento integral de los referentes en estudio, específicamente en la gestión documental. Razón por la cual en el LNDV se originó la necesidad de realizar procedimientos que permitieran la identificación de roles, divulgación de normatividades y el tipo de comunicación para establecer un sistema de control documental diferente a los establecidos por el ICA, con el fin de definir y regular los procesos documentales conforme a los requerimientos normativos vigentes, acatando las directrices y lineamientos de las normas de calidad internas del instituto. Esto evidenció la existencia de reprocesos, tanto de la parte institucional, como de la dependencia del LNDV.

La importancia de la gestión documental radica en que incluye importantes aspectos relacionados con el cambio cultural en la organización, asociados a los sistemas de gestión y relacionados directamente con la gestión del conocimiento, entre estos se destacan: plantear que la organización exija a su personal documentarse correctamente, tomar consciencia de la importancia de la documentación y que en los procesos de comunicación interna se haga referencia a la gestión documental (Álvarez, Castro y Peña, 2015). Comprende algo más que una readecuación y renovación de la tarea de los archiveros; "[...] puede considerarse como un proceso vital para toda organización debido a la magnitud que alcanza la producción de documentos, que permiten analizar y controlar sistemáticamente cómo la información registrada se crea, recibe, mantiene o utiliza" (Pacheco Zamudio, 2015, p. 107).

Para dar solución a las problemáticas documentales de la entidad, especialmente a la falta de articulación de la norma técnica del laboratorio con las normas de gestión de calidad, la presente investigación propone una metodología que permite la integración de las normas técnicas NTCGP 1000, NTC ISO/IEC 17025 y ISO 9001 en la gestión documental, partiendo del concepto de integración como un proceso que vincula diferentes sistemas de gestión estandarizados dentro de un único sistema de gestión con requisitos comunes y el objetivo de mejorar la satisfacción de las partes interesadas (Bernardo, Casadesus, Karapetrovic y Heras, 2009), lo que proporciona un elemento de integración que facilita la ejecución de los sistemas de gestión y unifica criterios en las etapas documentales para su adecuado control y gestión. Se plantea la hipótesis que afirma la existencia y compatibilidad entre los sistemas de gestión NTCGP 1000, ISO 9001:2008 y la NTC ISO 
17025 en la gestión documental, por medio de tres objetivos específicos: el primero consiste en identificar los aspectos críticos que afectan la gestión documental del LNDV ICA con respecto a los referenciales NTC ISO 9001, NTCGP 1000 e ISO/IEC 17025; el segundo pretende identificar los modelos de gestión que permiten la integración de los referenciales en mención para fortalecer la gestión documental del LNDV ICA, y el tercero busca estructurar una propuesta metodológica para la integración de los sistemas de gestión basada en las normas en estudio.

\section{METODOLOGÍA}

El diseño metodológico de esta investigación tiene un enfoque cualitativo. Para dar cumplimiento a los objetivos planteados se escogió como método el estudio descriptivo, con el fin de medir o recoger información de manera independiente o conjunta sobre los conceptos o las variables a las que se refiere el estudio, mas no indicar cómo se relacionan estas (Hernández Sampieri, Fernández Collado y Baptista Lucio, 2010).

Para la construcción de la propuesta se realizó inicialmente un grupo focal, en el cual el tema principal se enfocó a dar solución a la pregunta: ¿¿cuáles considera usted que son los aspectos críticos que afectan la gestión documental en el LNDV del ICA? Esta fue resuelta por parte de los funcionarios de la entidad, pertenecientes a las áreas de planeación, gestión documental y al grupo de buenas prácticas de laboratorio.

Esta información recolectada fue analizada y organizada por categorías nombradas según las respuestas en las dimensiones de conocimiento, formación y toma de conciencia, normas y leyes, planificación, responsabilidad y autoridad, gestión por procesos, recursos para el sostenimiento de los sistemas de gestión, procedimiento, control de documentos e implementación.
Las dimensiones de comunicación, mejora y desempeño se adicionaron posteriormente en la estructura de la propuesta metodológica, con el fin de llevar a cabo la ejecución del ciclo planificar-hacer-verificar-actuar.

El universo está conformado por un total de 17 personas pertenecientes a las áreas de planeación, gestión documental, grupo Buenas Prácticas de Laboratorio y coordinación del LNDV del ICA, dependencias que intervienen en el direccionamiento de la gestión documental. La distribución por personas pertenecientes a cada área se muestra en la tabla 1.

Tabla 1. Distribución de personas por áreas

\begin{tabular}{|l|c|}
\hline \multicolumn{1}{|c|}{ ÁREAS } & $\begin{array}{c}\text { NÚMERO } \\
\text { DE PERSONAS }\end{array}$ \\
\hline Área de planeación & 6 \\
\hline Área de gestión documental & 6 \\
\hline Grupo de Buenas Practicas de Laboratorio & 4 \\
\hline Coordinación del LNDV & 1 \\
\hline Total & 17 personas \\
\hline
\end{tabular}

Fuente: elaboración propia.

La muestra de trabajo se calculó en el programa estadístico $\mathrm{R}$, de acuerdo con la metodología de muestreo estratificado, ya que cada una de las áreas por investigar se puede identificar como un estrato. Se utilizaron fórmulas estadísticas para determinar el tamaño de la muestra en cada una de las áreas, dependiendo de la proporción. Los resultados obtenidos permiten concluir que una muestra significativa para las diferentes áreas es la que se presenta en la tabla 2.

Estos resultados representan una significancia estadística correspondiente al $10 \%$ de error y un nivel de confianza del $70 \%$, es decir, una potencia del 0,07. Del total de las áreas se seleccionaron siete personas, ya que están involucradas totalmente en el proceso de gestión documental; por lo tanto, son el total poblacional del estudio. 
Tabla 2. Muestra

\begin{tabular}{|c|c|}
\hline ÁREAS & $\begin{array}{c}\text { NÚMERO } \\
\text { DE PERSONAS }\end{array}$ \\
\hline Área de planeación & 2 \\
\hline Área de gestión documental & 1 \\
\hline Grupo de Buenas Practicas de Laboratorio & 3 \\
\hline Coordinación del LNDV & 1 \\
\hline Total & 7 personas \\
\hline
\end{tabular}

Fuente: elaboración propia.

Los instrumentos utilizados para la recolección de la información son los siguientes:

1) Lista de chequeo: con el fin de dar cumplimiento al objetivo número uno, este instrumento incluye los referenciales NTCGP 1000, ISO 9001:2008, NTC ISO 17025 y la Ley General de Archivo, con el fin de identificar el estado actual de cumplimiento del LNDV frente a cada referencial. Contiene 134 preguntas 0 afirmaciones redactadas según los resultados de una matriz de correlación realizada para identificar los numerales y apartados documentales de cada una de las normas y la Ley General de Archivo. La validación de este instrumento de investigación se realizó a través de expertos que evaluaron de acuerdo con los criterios establecidos en la tabla 3.

2) Encuesta: este instrumento tiene la finalidad de dar cumplimiento al objetivo número 1 , específicamente identificando los aspectos críticos que afectan la integración de los referenciales NTCGP 1000, ISO 9001:2008, NTC ISO 17025 y la Ley General de Archivo en el LNDV a través de 44 afirmaciones. Se realizó la validación por expertos, y en esta se incluyeron criterios presentados en la tabla 3.

Una vez aplicados los instrumentos de investigación y ejecutados los objetivos, se utilizaron las siguientes técnicas de procesamiento de la información: estadística descriptiva para identificar las posibles causas de la falta de articulación en los sistemas de gestión, análisis de contenido usando las herramientas de integración que permitan articular las normas en mención y elaboración de la propuesta metodológica, la cual permite organizar y jerarquizar las etapas y acciones por desarrollar para resolver las falencias detectadas.

Tabla 3. Criterios para la validación de instrumentos de investigación

\begin{tabular}{|l|l|}
\hline \multicolumn{1}{|c|}{ CRITERIOS } & \multicolumn{1}{c|}{ DEFINICIÓN } \\
\hline Coherencia & $\begin{array}{l}\text { Relación lógica entre dos cosas o entre las partes o elementos de algo, de modo } \\
\text { que no se produce contradicción ni oposición entre ellas. }\end{array}$ \\
\hline Claridad & $\begin{array}{l}\text { Está organizado adecuadamente el material lingüístico - palabras, oraciones- } \\
\text { para que el lector acceda a los contenidos sin mayores percances, a través de } \\
\text { una lectura fluida y perceptible. }\end{array}$ \\
\hline Pertinencia & $\begin{array}{l}\text { El contenido del ítem está directamente relacionado a cada dimensión, es } \\
\text { decir, se refiere o atañe a lo que se desea indagar para cada dimensión }\end{array}$ \\
\hline Suficiencia & $\begin{array}{l}\text { La información contenida en el ítem es suficiente para indagar acerca de lo que } \\
\text { se pretende de cada dimensión. }\end{array}$ \\
\hline
\end{tabular}

Fuente: elaboración propia. 


\section{RESULTADOS Y DISCUSIÓN}

\section{Aspectos críticos que afectan la gestión documental del LNDV del ICA con respecto a los referenciales NTC ISO 9001, NTCGP 1000 E ISO/IEC 17025}

Diagnóstico de gestión documental LNDV del ICA frente a los referentes NTC ISO 9001, NTCGP 1000 E ISO/IEG 17025.

De acuerdo con los resultados obtenidos en la lista de chequeo (presentados en la figura 1), el LNDV demostró un índice de cumplimiento de los referentes con un porcentaje alto. Esto indica que existen procesos y procedimientos escritos en pro del cumplimiento, tanto normativo como legal en la gestión documental, evidenciado principalmente en la política de gestión documental, la cual incluye los objetivos, las bases normativas y el ciclo de gestión documental. Además, para gestionar la documentación, la entidad cuenta con un gestor documental, que es una herramienta para el control de los documentos del sistema (caracterizaciones, manuales, procedimientos, métodos analíticos, instructivos, formas, etc.) y a través de esta se ha propuesto mejorar los procesos de administración documental. De esta manera, es una de las entidades públicas del país que se enfoca en potencializar la eficacia, eficiencia y efectividad de los servicios prestados a la sociedad a través de sistemas de gestión (Sáenz Urquijo y Serrano Turriago, 2012).

Cabe resaltar que con respecto a la Ley 594 de 2000, Ley General de Archivos, y según los resultados obtenidos en la lista de chequeo, ésta presenta el nivel de cumplimiento más bajo, con un número alto de preguntas sin contestar, lo que puede indicar que las personas que realizaron la calificación tienen desconocimiento de esta, lo que se correlaciona con los comentarios realizados por los funcionarios que participaron en el estudio. En este último se encontró que el conocimiento de este importante referente está centralizado únicamente en el área de gestión documental de la entidad. Lo anterior indica la necesidad de fortalecer la gestión del conocimiento en cuanto a la gestión documental de gran importancia por destacar en el Decreto 2609 del 14 de diciembre de 2012, por el cual se reglamenta el título $\mathrm{V}$ de la Ley 594 de 2000. Dicha ley dispone en su artículo $3^{\circ}$ que la gestión de documentos está asociada a la actividad administrativa del estado, al cumplimiento de las funciones y al desarrollo de los procesos de todas las entidades del estado; por lo tanto, es responsabilidad de los servidores y empleados, aplicar las normas que en esta materia establezca el Archivo General de la Nación (Decreto 2609, 2012).

De igual manera, se presenta un comportamiento similar con el referente ISO 17025, donde el conocimiento se centra en el grupo de buenas prácticas de laboratorio y la coordinación del LNDV; las demás áreas, planeación y gestión documental, manifiestan en la columna de observaciones un desconocimiento de la norma.

Correlacionando los resultados de la lista de chequeo y la encuesta de la figura 2, se evidencia que la mayoría de dimensiones fueron afectadas por el desconocimiento de alguno de los referentes de los funcionarios que participaron en el estudio, lo que perturbó directamente la dimensión de conocimiento. Estos funcionarios hacen observaciones específicas de desconocimiento con respecto a los aspectos de la gestión documental que no se manejan en su área. Las observaciones más relevantes en relación con esta dimensión están tienen que ver con los siguientes aspectos: no se tiene un concepto claro sobre integración de los sistemas de gestión para toda la institución, existe desconocimiento de los requisitos de documentación que establece la Ley 594 del 2000 y no existen suficientes directrices especificadas y documentadas en el LNDV acerca de la realización de backups; actualización de base de datos y organización de archivos electrónicos y físicos. 
Figura 1. Índice de cumplimiento por referente

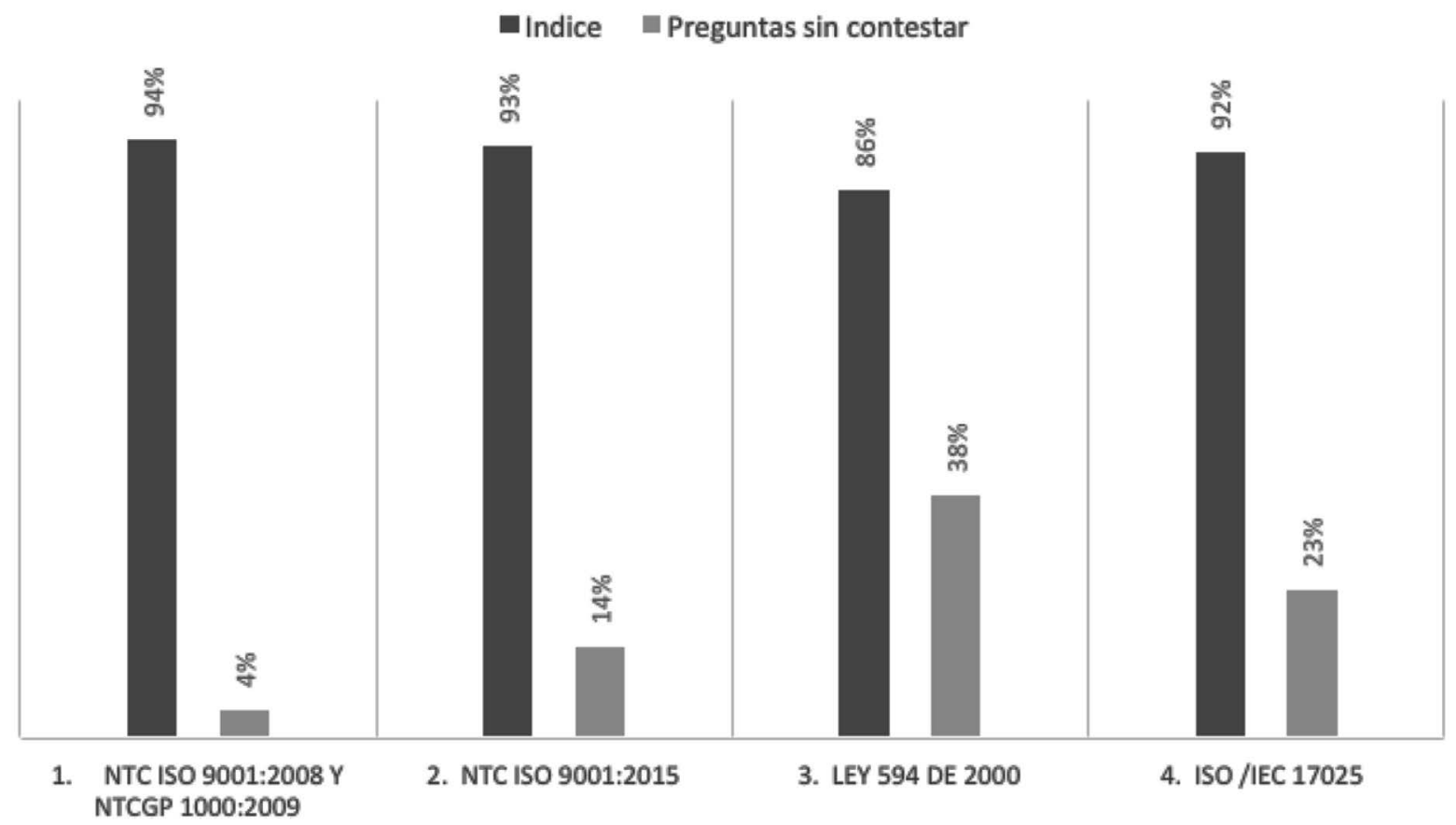

Fuente: elaboración propia.

Figura 2. Relación resultados de lista de chequeo y encuesta.

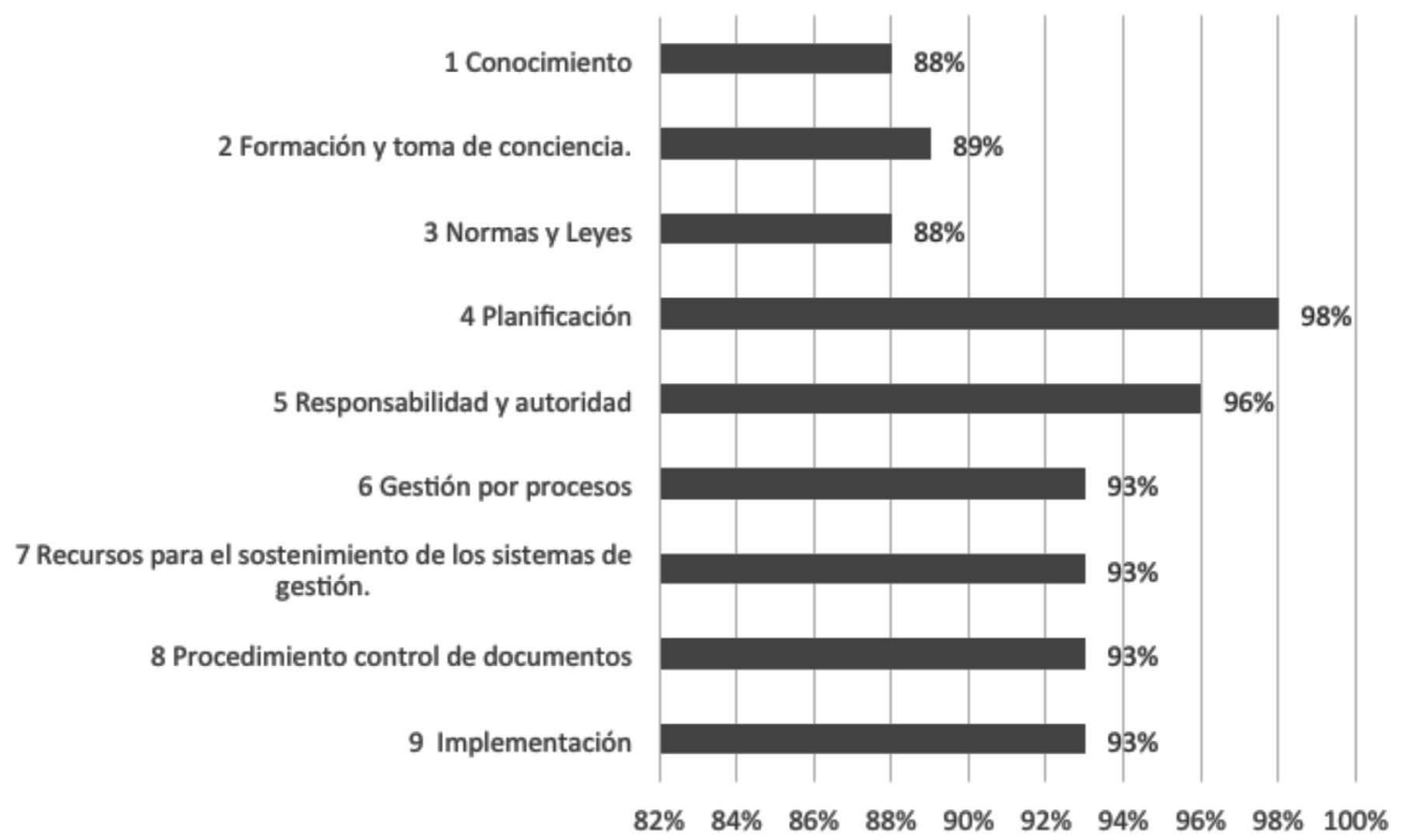

Fuente: elaboración propia. 
Lo anterior indica que la entidad presenta falencias frente al concepto que genera esta dimensión, en la cual para crear conocimiento nuevo en las organizaciones dicho concepto parte de los individuos, pero en el proceso de compartirlo es transformado en un conocimiento valioso para la entidad, como un todo que incluye etapas que implican crear, explorar, combinar y descubrir el conocimiento mediante el hacer (Bellinza, Sirtori y Mercado, 2011).

Según los resultados obtenidos, podemos deducir que en la entidad faltan mecanismos que permitan la integración de los requisitos de documentación de los referentes, los cuales son de obligatorio cumplimiento por parte de la entidad, y el facilitar la integración de estos contribuye a garantizar su ejecución, de manera tal que no existan reprocesos. Para lograr la integración de estos se debe seguir un proceso con el que se vinculen diferentes sistemas de gestión estandarizados dentro de un único sistema de gestión, con requisitos comunes y con el objetivo de mejorar la satisfacción de las partes interesadas (Bernardo et al., 2009).

En cuanto a la dimensión de planificación, se evidencia que en el instrumento lista de chequeo presenta un índice de cumplimiento alto en relación con el de la encuesta, que arrojo un índice de conformidad bajo, dado que la naturaleza de los instrumentos tiene una intención diferente, la lista de chequeo es dicotómica y la encuesta es de percepción. El ejercicio de planificación se realiza de manera estratégica integrando e involucrando a todos los grupos para que asuman un papel significativo en el desarrollo y la entrega de los documentos, de tal forma que todos participan en conjunto como un gran equipo y no como una serie de expertos individuales.

Así es como en la actualidad, en el LNDV solo se elaboran documentos específicos del laboratorio, y con esto se presenta una debilidad importante donde cada responsable del proceso y dueño de la documentación la diseña desde la operación estructural (áreas o dependencias de la entidad), mas no desde el enfoque basado en procesos; aspecto que indica que no existe una interacción de estos desde la parte documental. Es necesario implementar estrategias encaminadas a plantear que la organización exija a su personal documentarse correctamente, tomar conciencia de la importancia de la documentación y que en los procesos de comunicación interna se haga referencia a la gestión documental (Álvarez et al., 2015).

Teniendo en cuenta los resultados obtenidos en la dimensión de recursos para el sostenimiento de los sistemas de gestión - la cual demostró en la lista de chequeo un índice de cumplimiento alto en relación con la encuesta, que presentó un índice de conformidad bajo - se puede identificar que existen necesidades de recursos necesarios para el sostenimiento de la parte tangible y la intangible (equipos, hardware, software). De esta manera, según los resultados, en el LNDV los espacios físicos destinados a la conservación de archivos son insuficientes y con los que cuenta son inadecuados y no cumplen con lo pertinente a la Ley 594 de 2000. De esta manera se identifica que en los sistemas de gestión implementados no se ha dado el grado de importancia al cumplimiento en los numerales relacionados con recursos; por lo tanto, es necesario replantear y tener en cuenta que la implementación de un sistema de gestión es un conjunto de procesos interconectados que comparte recursos humanos, información, materiales, infraestructuras y recursos financieros, a fin de lograr los objetivos vinculados con la satisfacción de una gran variedad de stakeholders (Bernardo et al., 2009).

La gestión documental puede considerarse como un proceso vital para toda organización, debido a la 
magnitud que alcanza la producción de documentos, la relación con la gestión de la información y la gestión del conocimiento; permite, además, analizar y controlar sistemáticamente de qué manera la información registrada se crea, recibe, mantiene o utiliza (Risso, 2012), actividades que el laboratorio necesita para resolver sus actuales problemas en la gestión documental.

De acuerdo con lo anterior, es necesario tener en cuenta la etapa de implementación de los sistemas de gestión a través de mecanismos administrativos como estrategias, procedimientos, presupuestos, entre otros. Estos deben estar sistemáticamente relacionados y directamente vinculados con lo instaurado en la planificación (Vergara, 2009), lo que permite poner en práctica medidas y métodos para concretar alguna actividad, plan o misión y así desarrollar la gestión documental en un sistema de gestión, ya que finalmente lo que se encuentra documentado es lo que se debe hacer en la realidad (Guerra Bretaña, Meizoso Valdés y Roque González, 2016).

En consecuencia, en el LNDV existe la necesidad de generar cambios en la gestión documental; sin embargo, esto es un análisis que debe hacerse a la luz de la metodología de documentación adoptada por la entidad, el comité de coordinación del sistema de control interno y el comité de gestión de la calidad. Lo anterior se hace definiendo claramente las responsabilidades en materia documental, para toda la institución y sin permitir que las necesidades de las áreas o dependencias se conviertan en brechas; de esta forma, se genera la gestión del cambio y se correlaciona directamente con las diferentes investigaciones de implementación de sistemas de gestión, en las cuales se ha concluido que la NTCGP 1000: 2009 e ISO 9001: 2008 se comportan como modelo de gestión del cambio (Santander Benavides, 2012).

\section{Modelos de gestión para la inteǵración de los referenciales NTC ISO 9001, NTCGP 1000 E ISO/IEC 17025}

De acuerdo con el análisis de contenido realizado para seleccionar la metodología de integración apropiada — en cada dimensión se establecieron aspectos generales, en común o de divergencia de los modelos de integración estudiados-, el grupo de investigación llegó a la conclusión de que el modelo de integración que más se ajusta a la entidad, a las normas y leyes en estudio, y que desarrolla de manera detallada las dimensiones seleccionadas (conocimiento, formación y toma de conciencia, normas y leyes, planificación, responsabilidad y autoridad, gestión por procesos, recursos para el sostenimiento de los sistemas de gestión, procedimiento control de documentos e implementación) es la estructura de alto nivel, debido a que los demás modelos son iniciativas internacionales no actualizadas para los sistemas de gestión, lo más importante, requieren cierto nivel de integración implementado, condición que el LNDV no cumple.

En la actualidad, la organización internacional de estandarización ISO, con la estructura de alto nivel, pretende fomentar la compatibilidad entre las diversas normas de sistemas de gestión para facilitar su integración y su implementación por las organizaciones, en búsqueda de garantizar la calidad de la producción de sus propias normas: textos consistentes que permanecen aplicables a organizaciones de todos los tamaños, en todos los sectores y operando en todas las culturas (Hernández et al., 2016).

De esta forma, a través de una encuesta realizada por ISO se establecen pautas para la conformación de la nueva estructura. El análisis de resultados de la encuesta hecha en la web de los usuarios actuales y potenciales de la norma ISO 9001, llevado a cabo en diez 
idiomas con un total de 11.722 respuestas de 122 países, indica que aunque había satisfacción significativa con la versión 2008 de la norma, la mayoría de los usuarios consideró apropiado realizar una revisión. A raíz de esta encuesta efectuada a los usuarios de ISO, el comité técnico TG 176/sub comité 2 decidió que la revisión era conveniente y definió los siguientes objetivos para mantener su relevancia en el mercado actual y futuro (Palmes, 2015).

1. Integración con otros sistemas de gestión.

2. Proporcionar un enfoque integrado de la gestión organizacional.

3. Reflejar los entornos cada vez más complejos donde operan las organizaciones.

4. Mejorar la capacidad de la organización para satisfacer a sus clientes.

La estructura de alto nivel proporciona un marco de trabajo que garantiza una salida adecuada del proceso de desarrollo de la norma ISO, la cual permitirá al LNDV del ICA iniciar su proceso de certificación bajo la norma ISO 9001:2015 y estar al tanto de las actualizaciones normativas.

\section{Propuesta metodológica para la integración de los sistemas de gestión basado en los referenciales NTC ISO 9001, NTCGP 1000 E ISO/IEC 17025}

Teniendo en cuenta los resultados de la aplicación de los instrumentos de investigación y el análisis de contenido, la construcción de la metodología que permitirá la integración de los sistemas de gestión basados en los referenciales NTC ISO 9001, NTCGP 1000 e ISO/IEC 17025 tiene como objetivo llegar a organizar y jerarquizar las etapas y acciones por desarrollar, para resolver las brechas detectadas como aspectos críticos, que afectan la gestión documental en cada una de las dimensiones.

Estos resultados se analizaron a través de una matriz denominada "Matriz de identificación de aspectos críticos y sus posibles soluciones" (anexo 2), realizando un cruce de información de los análisis de resultados obtenidos.

Una vez hecha la matriz se procedió a elaborar las diferentes herramientas que permitieron construir la propuesta metodológica. Es importante señalar que el resultado de esta investigación es coherente con cualquier estudio descriptivo y su intención es proporcionar a la entidad un "paso a paso" de cómo integrar la gestión documental de las normas con las cuales está certificada, incluyendo algunos referentes de obligatorio cumplimiento.

Las dimensiones propuestas se identificaron al inicio de la investigación y en la recolección de datos; sin embargo, en la construcción de la presente propuesta y con el fin de estar acorde al modelo de integración seleccionado (estructura de alto nivel), surgió la necesidad de incluir las siguientes dimensiones:

- Desempeño.

- Mejora.

- Comunicación.

Además, se incluyó el contexto como un componente de partida para dar a conocer las partes interesadas y los factores internos y externos que pueden afectar las dimensiones de la propuesta metodológica (anexo 3).

Este modelo aplica la metodología conocida como planificar-hacer-verificar-actuar, en el cual el planificar y el hacer se encuentran asociados a la creación y utilización de documentos, y el verificar y actuar se relacionan con la utilización de los registros. 
Figura 3. Esquema de la propuesta metodológica para la integración de un sistema de gestión basado en los referenciales NTC ISO 9001, NTCGP 1000 e ISO/IEC 17025 en la gestión documental del LNDV ICA.

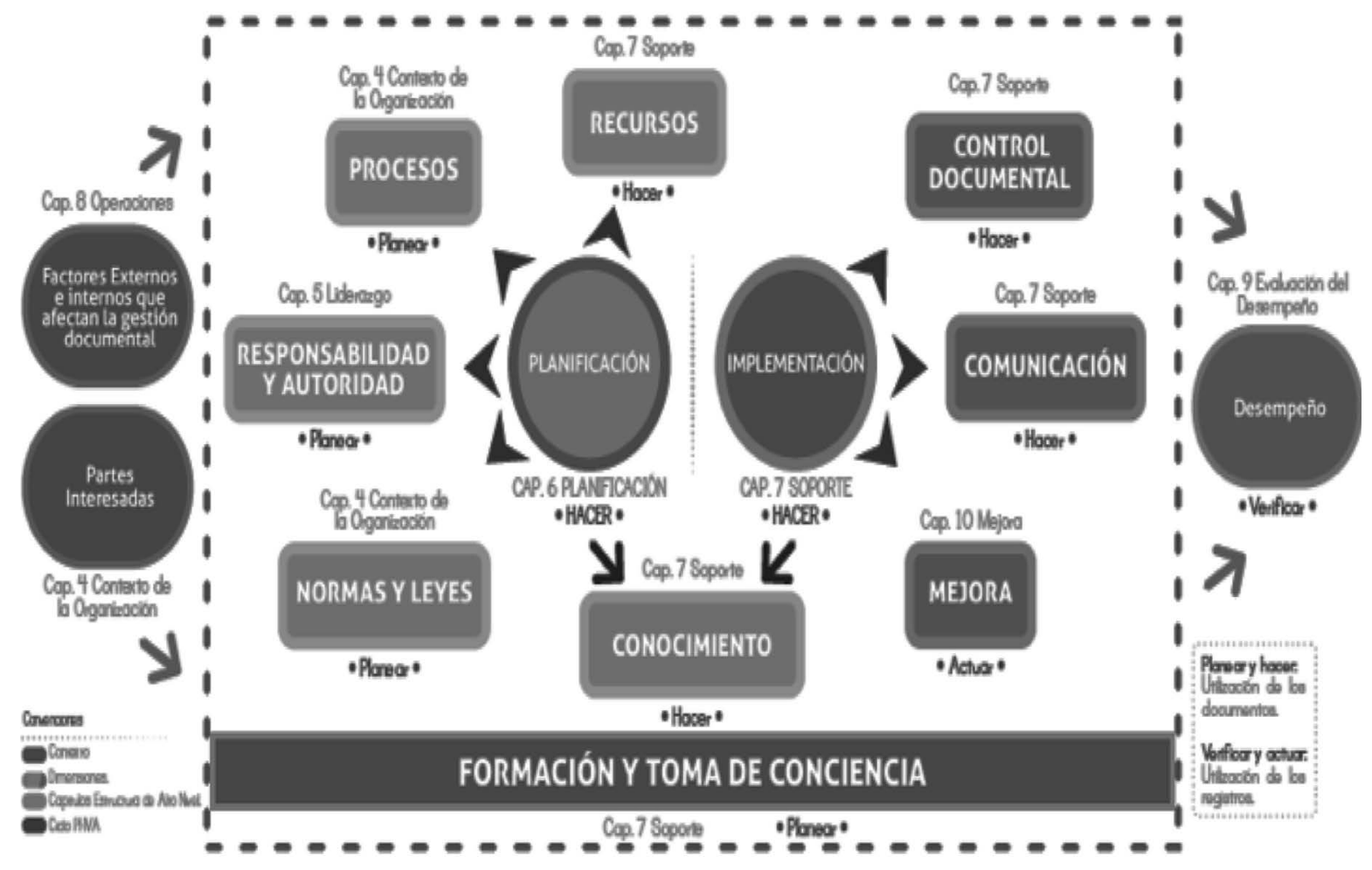

Fuente: elaboración propia.

\section{CONCLUSIONES}

Los instrumentos construidos y utilizados para la identificación de los aspectos críticos que afectan la gestión documental del LNDV del ICA, con respecto a los referenciales, fueron herramientas asertivas e innovadoras en la entidad, puesto que además de contar con un diagnóstico del índice de cumplimiento normativo a través de la lista de chequeo, se complementó con la encuesta. Esta última determinó el índice de percepción de los funcionarios con respecto a las falencias identificadas desde el planteamiento del problema y que constituyó la base para la proposición de estrategias y herramientas en la propuesta metodológica.
Por otro lado, los aspectos críticos identificados ocasionan falencias en las ejecuciones que corresponden a la gestión documental y pertenecen a los diferentes procesos estructurales de los sistemas de gestión, en los cuales sus causas y consecuencias están asociadas a los componentes normativos identificados como dimensiones.

En cuanto a la construcción de la propuesta metodológica para la integración de los sistemas de gestión, basada en los referenciales de la gestión documental del LNDV del ICA, se evidenció que las entidades que implementan sistemas de gestión requieren estrategias que les permitan adoptar metodologías para los 
distintos procesos. La construcción de esta propuesta, y las herramientas que la conforman, se basó en la identificación de las dimensiones que le permitan a la entidad un mejor control de las actividades, el logro de los objetivos definidos para cada etapa de la gestión documental, de una manera efectiva y, ante todo, la adopción de la cultura de la integración para facilitar la gestión del día a día.

La investigación concluye que la hipótesis planteada sobre la existencia de compatibilidad entre los sistemas de gestión NTCGP 1000, ISO 9001:2008 y la NTC ISO 17025 en la gestión documental es verdadera, debido a que en la construcción de la matriz de correlación se evidenció que los requisitos documentales para cada una de las normas se pueden integrar, a pesar de que la norma NTC ISO 17025 incluye capítulos específicos referidos al que hacer de los laboratorios. Pero estos no la hacen incompatible con los otros sistemas de gestión en los cuales los capítulos referidos a la gestión documental son semejantes y facilitan su integración.

Finalmente, se puede concluir que el objetivo general de la investigación se alcanzó satisfactoriamente, pues se construyó una propuesta metodológica que ha suministrado un primer aporte para los laboratorios de diagnóstico veterinario que quieran poner en práctica la integración de los referentes en gestión documental, con el fin de respaldar los procesos y fortalecer el sistema de gestión de calidad

Este estudio podrá ser usado en futuras investigaciones para ser aplicado en otras entidades, que permitan validar la metodología con una muestra representativa y posteriormente realizar mediciones sobre el nivel de integración de los sistemas de gestión de calidad en la gestión documental.

Dependiendo del tamaño de la organización y sus sistemas de gestión, es necesario empezar con la aplicación de la propuesta de una manera sencilla, entendible y coherente, con el fin de motivar al personal en todos los niveles de la organización, puesto que adoptar una propuesta metodológica representa un cambio en la cultura organizacional que requiere constancia y dedicación para que se lleve a cabo de manera exitosa.

\section{REFERENCIAS}

Álvarez, M., Castro, M. y Peña, G. (2015). Gestión del conocimiento: una perspectiva desde la gestión de la calidad y la gestión documental. Signos, 5(2), 101-118.

Bernardo, M., Casadesus, M., Karapetrovic, S. y Heñras, I. (2009). How integrated are environmental, quality and other standardized management systems? An empirical study. Journal of cleaner production, 17(8), 742-750.

Bellinza, M., Barrera, N. G., Sirtori, S. C. y Mercado, W. R. (2011). Gestión del conocimiento: Aproximaciones teóricas. Clío América, 5(10), 257-271.

Ministerio de Cultura de Colombia. (14 de diciembre de 2012). "Decreto 2609". Diario Oficial de la Republica de Colombia. Bogota, Colombia.

Guerra Bretaña, R. M., Meizoso Valdés, M. D. C. y Roque González, R. (2015). Normalización y aplicación de los principios de gestión de la calidad en la actividad archivística. Revista Habanera de Ciencias Médicas, 14(4), 527-535.

Hernandez, Y. (2016). Diseño de un esquema para la certificación de la norma NTC 9001: 2015 con base en los lineamientos de las normas ISO/IEC 17021: 2011 y ISO/IEC TS 17021-3, en Basc Bogotá Colombia para los sectores vigilancia y seguridad, transportador de 
Propuesta metodológica para la integración de un sistema de gestión documental basado en los referenciales NTC ISO 9001, NTCGP 1000 e ISO/IEC 17025

carretera, importador y exportador. Trabajo de grado de especialización. Escuela Colombiana de Ingeniería Julio Garavito, Bogotá, Colombia.

Hernández R., Fernández C. y Baptista P. (2010). Metodología de la Investigación. México: Mc. Graw Hill.

Pacheco, M. (2015). El control del sistema de gestión documental de los fondos municipales como mecanismo para asegurar la conservación del patrimonio documental. Comma, 2014(1-2), 105-111.

Palmes, P. C. (2015). ISO 9001: 2015 transition starts with top management. Recuperado de https://secure.asq.org/perl/msg.pl?prvurl=http://asq.org/asq. org/2015/05/standards/iso-9001-2015-transitionstarts-with-top-management.pdf.

Risso, V. (2012). Aproximación teórica a la relación entre los términos gestión documental, gestión de información y gestión del conocimiento. Revista Española de Documentación Científica, 35(4), 531.

Santander, F. (2012). La responsabilidad social, más allá de la gestión de la calidad en los gobiernos locales de Colombia. Signos, 4(2), 51-69.

Sáenz, J. y Serrano, I. (2012). El sistema de gestión de la calidad como modelo de cambio organizacional en el sector público colombiano. Signos, 4(1), 13-53.

Vergara, G. (2009). Qué es un sistema de gestión. Mejora tu gestión. Recuperado de http://implementacionsig.com/index.php/23-noticiac/28-que-es -un-sistema-de-gestion

Villaquirán, V. y Nieto, Y. (2016). Sistema de gestión de calidad, factor importante para el desarrollo laboral del colaborador de Unisalud Palmira. Colección Académica de Ciencias Estratégicas, 3(1), 41-60. 